\section{Radiation Protection Capability of Operating Room Personnel: Development and Psychometric Properties of a Questionnaire}

\author{
Zinat Mohebbi ${ }^{1 \oplus}$, Roya Ershadpoor ${ }^{2}$, Mohsen Ostovari³, \\ Mahnaz Rakhshan ${ }^{*} \odot$
}

\begin{abstract}
Background: Radiation protection is an important principle in some wards of the hospital such as radiology, catheterization laboratory and operating room. Due to the increasing use of radiation in the operating room, there is a need to design an accurate and appropriate tool to evaluate the radiation protection capability of operating room personnel.
\end{abstract}

Objective: This study aims to test the psychometric properties of a questionnaire on radiation protection capability.

Material and Methods: This cross-sectional study was conducted in two stages. The first stage was designing items based on the review of available literature, and the second stage was measuring the validity and reliability of the questionnaire using face validity and content validity Content Validity Index (CVI) and Content Validity Ratio (CVR). Then the questionnaire was filled out by 200 operating room nurses to evaluate the construct validity by Principal Component Analysis method. Reliability of the questionnaire was evaluated by test-retest and Cronbach's alpha analysis method.

Results: Due to the results, test-retest correlation coefficient was 0.912 , and Cronbach's alpha coefficient was 0.824 , indicating a desirable internal consistency.

Conclusion: This study introduces a valid and reliable questionnaire for evaluating the radiation protection capability of operating room nurses.

Citation: Mohebbi Z, Ershadpoor R, Ostovari M, Rakhshan M. Radiation Protection Capability of Operating Room Personnel: Development and Psychometric Properties of a Questionnaire. J Biomed Phys Eng. 2021;11(5):603-612. doi: 10.31661/jbpe.v0i0.2008-1161.

\section{Keywords}

Radiation Protection, Questionnaire; Operating Room Nursing, Psychometrics, lonizing Radiation, Operating Theatre

\section{Introduction}

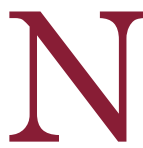

owadays, radiation is widely used in operating rooms [1]. In addition to the practical benefits of radiation, its side effects should be considered since the ionizing radiation increases the risk of DNA damage and cancer in nurses and other operating room personnel [2-4].

The most commonly used radiation-generating device in the operating room is C-arm or fluoroscope, which is used in a variety of surgeries such as orthopaedics, urology, neurosurgery, and angiography [5]. The
${ }^{1} \mathrm{PhD}$, Department of Nursing, School of Nurs-

ing and Midwifery, Shiraz

University of Medical

Sciences, Shiraz, Iran

${ }^{2} \mathrm{MSc}$, Student research

committee, Shiraz

University of Medical Sci-

ences, Shiraz, Iran

${ }^{3} \mathrm{PhD}$, Department of

Medical Physics and

Engineering, School of

Medicine, Shiraz Univer-

sity of Medical Sciences,

Shiraz, Iran

*Corresponding author:

Mahnaz Rakhshan

School of Nursing

and Midwifery, Shiraz

University of Medical Sci-

ences, Zand Blv, Namazi

Square, Shiraz, Iran

E-mail: mzrakhshan@ ymail.com

Received: 17 August 2020 Accepted: 20 October 2020 
use of radiation shield significantly reduces the radiation dose received by nurses, surgeons and patients [6-8]. Awareness of the principles of radiation protection and their observance by nurses working in the operating room has a significant role in reducing the risks of ionizing radiation. [1, 9-12]. Therefore, a comprehensive tool evaluating all aspects of radiation protection in the operating room personnel could be useful [13].

The Australian Institute of Radiology (AIS) defines radiographers' 'professional capability' as the application of professional knowledge and skill to perform a desired function. This professional capability includes several skills such as knowledge, professional communication skills, teamwork skill, risk management, safety, technical skills and critical thinking skills in the field of working with Xrays [14]. Therefore, radiation protection capability is a desirable concept to evaluate the knowledge, attitude, practice and professional commitment of nurses and surgeons in risk management and protection of personnel and patients against radiation [13-15].

Literature review in databases of Web of Science, PubMed and Scopus and so on indicated that there were few questionnaires, specifically designed for measuring radiation protection capability in the operating room field, and the available questionnaires are intended for measuring limited dimensions such as knowledge of radiation protection [1, 9-12]. Also, few studies were about radiation protection capability while considering the patient safety against radiation $[13,16]$. Moreover, the most of the available questionnaires about radiation protection were designed to be used in radiology wards and they are not appropriate and adequate to be used in the operating room, where the sterility rules and some other principles influence radiation protection acts [1725]. Thus, working situation and professional duties related to the application of radiation in the operating room ward are different from radiology ward, and are not even similar in the type of radiation generating devices, physical environment, and individuals' knowledge about physics of radiation [7, 8, 26, 27].

Therefore, an operating room-specific radiation protection questionnaire should be designed.

Hence, given the increasing use of radiation in the operating room, it is important that operating room personnel have the capability to protect against radiation. In this regard, the designing and psychometrics of a valid and relevant tool can be useful in acquiring knowledge and understanding of this matter. Therefore, the aim of this study was to design a questionnaire about radiation protection capability of operating room nurses and surgeons.

\section{Material and Methods}

\section{Design, setting and subjects}

This cross-sectional study was conducted in two stages. In first stage, the items were designed based on the review of available literature, and in second stage, the validity (face validity and content validity) of the questionnaire was evaluated. Then construct validity was evaluated by Principal Component Analysis method. Reliability of the questionnaire was evaluated by test-retest and Cronbach's alpha analysis method.

In stage one, the items were extracted through literature review, including the national and international guidelines [7, 8, 26], books $[27,28]$ and related articles within the past 10 years [1, 9-13, 17-25]. Then the selected items were evaluated by the panel of experts. The panel of experts included several experienced specialists in the field of operating room nursing, psychometrics, radiology and nuclear medicine as well as some experienced radiographers in the operating room, all of whom were familiar with the concept of radiation protection. Eventually, the final draft with 120 items was approved. The considered dimensions of the questionnaire include knowledge, attitude, practice and professional 
Questionnaire of Radiation Protection Capability

commitments. A 5-point Likert scale (never, rarely, occasionally, usually, and always) was used to score the items.

\section{Data analysis \\ validity}

To evaluate the validity of the questionnaire, both face validity and content validity (Content Validity Ratio (CVR) and Content Validity Index (CVI)) were tested using qualitative and quantitative approaches. Construct validity was also determined by Principal Component Analysis method.

Face validity

Face validity was confirmed by quantitative and qualitative methods. Qualitative face validity was evaluated by a panel of 6 experts. Each expert checked the questionnaire and presented their opinions about the difficulty, relevancy, comprehensibility and ambiguity of each item.

In quantitative validity method, the importance of each item was evaluated by the 6 experts (concept and psychometric) and 6 operating room nurses. The importance of each item was evaluated using a five-point Likert scale. Finally, if the impact score of the item was equal or greater than 1.5 , the item was kept for the following steps [29].

Content validity

Qualitative and quantitative methods were used to determine the content validity. This phase was performed by 8 experts. In the qualitative content validity, each expert checked the questionnaire and presented their opinions about the grammar of items, the use of appropriate words and placement of items in the proper domain.

Quantitative content validity was evaluated by using Content Validity Ratio (CVR) and Content Validity Index (CVI). CVR was evaluated to check the necessity of the items by a three-point Likert scale. According to Lawshe [30], a score, which was higher than 0.75 , was significant.

CVI was evaluated to check the relevancy, simplicity and clarity of the items. In CVI survey based on the Waltz Content Validity Index, a higher score of 0.79 was recommended for the acceptance of the items [31].

\section{Construct validity}

The questionnaire was filled out by 200 operating room nurses using census method for evaluating the construct validity. KaiserMeyer-Olkin (KMO) measure of sampling adequacy and Bartlett's test of sphericity were investigated to test the correlation between the items of the questionnaire. KMO score, which was higher than 0.7 , was significant.

Principal Component Analysis (PCA) was used to integrate the correlated items and to obtain the true dimensions of the questionnaire. Item reduction and contribution of items to the instrument were measured by factor loading and "alpha if item deleted" method [32]. SPSS Version 25 was used for the analysis of the data.

\section{Reliability}

Reliability with internal consistency approach were checked through the Cronbach's alpha and test-retest analysis amongst 200 operating room nurses. Test-retest approach was used to measure the stability of the questionnaire among 26 operating room nurses. Based on the recommendations [33], the retest was taken 14 days after the primary test.

The minimum and maximum score of clinical capability were 27 and 135, respectively. In addition, good, moderate, and weak classes of radiographers were determined based on achieving $\geq 75 \%, 50 \%-75 \%$, and $<50 \%$ of the total score, respectively. Table 1 shows the cut off points of the scale.

Table 1: Cut off points of clinical capability and its subscales.

Scales Clinical capability knowledge Attitude practice

\begin{tabular}{c}
\hline Good $>108>40>28>40$ \\
\hline Moderate 81-108 30-40 21-28 30-40 \\
\hline Weak $<81<30<21<30$
\end{tabular}


Results

\section{Participants' demographics}

200 operating room nurses were tested in order to evaluate the construct validity and reliability of the designed questionnaire. The mean age of the participants was $33.34 \pm 8.09$ years and length of work experience was $9 \pm$ 7.37 years, respectively. Table 2 shows the result of participants' demographic information.

\section{Validity}

\section{Face validity}

In qualitative face validity, the pool of items was checked by the experts' panel and inappropriate and extra items were removed from the questionnaire. In doing so, the items were reduced from 120 to 63 . In quantitative face validity, the items with the score of less than 1.5 were removed from the questionnaire; hence, the items were reduced from 63 to 40 . The mean score of quantitative face validity was 3.024, respectively.

\section{Content validity}

After evaluating the qualitative content validity, 1 item was removed from knowledge dimension and 3 items from attitude dimension. After evaluating the quantitative content validity, 1 item was removed from attitude dimension, 2 items were removed from practice dimension and 1 item was removed from professional commitment dimension. Also, one item in attitude dimension and one item in practice dimension were changed. Finally, the present questionnaire consisted of 32 items, of which 10 items were in knowledge dimension, 7 items in attitude dimension, 10 items in practice dimension and 5 items in professional commitment dimension. The average content validity index of the questionnaire (S-CVI/ Ave) was 0.97 and the average Content Validity Ratio (s-CVR/ Ave) was 0.93, respectively. Table 3 shows the results of face validity, CVR and CVI.

Construct validity

Kaiser-Meyer-Olkin measure of sampling
Table 2: Demographic characteristics of the participants in the reliability part of the study.

\begin{tabular}{ccc} 
Variable & Frequency & Percent \\
\hline \multicolumn{3}{c}{ Age } \\
\hline Under 30 & 87 & 44.6 \\
\hline $30-40$ & 73 & 37.4 \\
\hline Above 40 & 35 & 17.9 \\
\hline \multicolumn{3}{c}{ Gender } \\
\hline Men & 111 \\
\hline Women & 88 \\
\hline \multicolumn{3}{c}{ Marital status } \\
\hline Single & 80.8 \\
\hline Married & 120 \\
\hline \multicolumn{3}{c}{ Education level } \\
\hline Associate degree & 26 & 40 \\
\hline Bachelor Degree & 132 \\
\hline Master Degree and \\
physician
\end{tabular}

\begin{tabular}{ccc}
\hline \multicolumn{3}{c}{ Occupation } \\
\hline Operating room nurse & 168 & 84 \\
\hline \multicolumn{3}{c}{ Type of hospital } \\
\hline Surgeon & 145 & 16 \\
\hline State hospital & 55 \\
\hline Private hospital & 27.5 \\
\hline \multicolumn{3}{c}{ Shift type } \\
\hline Fixed shift & 46 \\
\hline Shift in circulation & 152 \\
\hline \multicolumn{3}{c}{ Work field } \\
\hline Orthopaedic & 89 \\
\hline Urologic & 46 \\
\hline Type of employment \\
\hline Official & 76 \\
\hline Unofficial & 122 \\
\hline Working Experience as operating room personne \\
\hline under 5 years & 76 \\
\hline above 5 years & 112 \\
\hline Working Experience as operating room radiographer \\
\hline under 5 years & 125 \\
\hline above 5 years & 61 \\
\hline
\end{tabular}


Table 3: The results of quantitative part of face validity and content validity.

Items of the questionnaire

\begin{tabular}{lccc}
\hline 1- I know the effects of radiation exposure (at a higher dose than the standard level) on human health. & 4.16 & 1 & 1 \\
\hline 2- I know about As Low As Reasonably Achievable (ALARA) principle. & 2.75 & 0.85 & 0.85 \\
\hline 3- I know the standard distance from the X-ray generator. & 4.14 & 1 & 1 \\
\hline 4- I know the correct locations of film badge, depending on the type of imaging. & 3.78 & 0.9 & 0.85 \\
\hline 5- I know the standard method of keeping film badge safe after work. & 4.14 & 0.75 & 1 \\
\hline 6- I Know the ten day rule about women's radiography (because of the possibility of pregnancy). & 2.07 & 0.95 & 1 \\
\hline 7- I know that pulsed fluoroscopy can reduce the personnel and patient's radiation exposure. & 3.2 & 1 & 1 \\
\hline $\begin{array}{l}\text { 8- I know that scattered radiation from the patient's body is the most important source of personnel's } \\
\text { radiation exposure. }\end{array}$ & 4.6 & 1 & 1 \\
\hline $\begin{array}{l}\text { 9- I know the potencies of C-arm device in reducing the patients and personnel's radiation exposure. } \\
\text { 10- I know that collimation or limiting the field of radiation to the desired level reduces the patients and } \\
\text { personnel's radiation exposure. }\end{array}$ & 2.36 & \multirow{2}{*}{1} & \multirow{2}{*}{0.95} \\
\hline
\end{tabular}

11- The width of the operating room space around the under irradiation patient is essential (for the observance of standard distance).

12- Existence of safety instructions and radiation warning posters is essential in the operating room.

13- The use of film badge is essential for all the personnel exposed to radiation in the operating room.

14- Regular health check and blood test are required for operating room personnel.

15- Using lead gloves is essential if personnel's hands are close to the source of radiation.

16- When using a portable X-ray machine in the operating room, the use of lead Paravan is also essential, in addition to other safeguards.

17- It is essential to use a hazard warning light above the door of under radiation operating room.

18- I assure female patients' possibility of pregnancy before the surgery.

19- I record the correct frequency and duration of the radiation in the patient's file.

20- I regard the standard distance of the X-ray generator to the patient's skin.

21- In vertical imaging, I regard the correct positioning with the image intensifier above and X-ray generator under the patient.

22- In horizontal imaging, I stand on the side of image intensifier.

23- I use my personal dosimeter.

24- I use lead aprons, despite being heavy, for my personal protection.

25- I use thyroid shield during radiation exposure.

26- I place genital shield for the patient under radiation.

27- I place thyroid shield for the patient under radiation.

28- Patient's radiation protection is important for me.

29- I cooperate with other operating room personnel in the field of radiation protection.

30- I use standard guidelines and scientific texts about radiation protection.

31- I am responsive for my radiation protection act.

32- I participate in radiation protection training courses periodically.

IS: Item Impact score, CVR: Content validity ratio, CVI: Content Validity Index

IS CVR CVI

$\begin{array}{lll}4.7 & 1 & 1\end{array}$

\begin{tabular}{ccc}
4.05 & 0.85 & 1 \\
\hline 4.41 & 1 & 1 \\
\hline 4.9 & 1 & 1 \\
\hline 3.52 & 0.8 & 1
\end{tabular}

\section{$\begin{array}{lll}4.9 & 0.75 & 0.85\end{array}$}

\begin{tabular}{ccc}
4.9 & 0.75 & 1 \\
\hline 4.9 & 1 & 1 \\
\hline 2.04 & 1 & 1 \\
\hline 4.7 & 1 & 1 \\
\hline
\end{tabular}

$\begin{array}{lll}2.8 & 1 & 1\end{array}$

\begin{tabular}{|ccc}
\hline 3.44 & 0.85 & 0.85 \\
\hline 2.31 & 1 & 1 \\
\hline 5 & 1 & 1 \\
\hline 5 & 0.95 & 1 \\
\hline 4.9 & 1 & 1 \\
\hline 5 & 1 & 1 \\
\hline 5 & 1 & 1 \\
\hline 3.28 & 0.8 & 1 \\
\hline 4.8 & 0.95 & 1 \\
\hline 4.9 & 0.75 & 1 \\
\hline 4.05 & 1 & 1 \\
\hline
\end{tabular}


adequacy was 0.837 ; Bartlett's test of sphericity was 3438.304 ( $\mathrm{P}$ value $=0.0001)$, respectively.

The results of PCA (Table 4) showed that the factor loading of most of the items was the highest value in its considered dimension. According to Table 4 , item 1 to 10 had the highest value in knowledge dimension, and item 11 to 17 had the highest value in attitude dimension. Items 19, 20, 22, 26 and 27 had the highest value in practice dimension; however, no item had a high value in professional commitment dimension. Finally, according to factor loading of items, 5 items considered in profession-

Table 4: Principal component analysis of clinical capability dimensions.

\begin{tabular}{|c|c|c|c|c|}
\hline Items of the questionnaire & Knowledge & Attitude & Practice & Professional commitment \\
\hline Q1 & 0.687 & -0.118 & -0.283 & -0.164 \\
\hline Q2 & 0.712 & -0.169 & -0.168 & -0.100 \\
\hline Q3 & 0.680 & -0.029 & -0.290 & -0.085 \\
\hline Q4 & 0.775 & 0.012 & -0.223 & -0.174 \\
\hline Q5 & 0.747 & -0.055 & -0.264 & -0.074 \\
\hline Q6 & 0.711 & -0.176 & -0.142 & 0.061 \\
\hline Q7 & 0.683 & -0.129 & -0.294 & -0.106 \\
\hline Q8 & 0.767 & -0.121 & -0.166 & -0.117 \\
\hline Q9 & 0.761 & -0.141 & -0.234 & -0.107 \\
\hline Q10 & 0.731 & -0.079 & -0.167 & -0.150 \\
\hline Q11 & -0.048 & 0.705 & -0.322 & -0.120 \\
\hline Q12 & -0.053 & 0.727 & -0.297 & -0.026 \\
\hline Q13 & 0.001 & 0.705 & -0.355 & -0.068 \\
\hline Q14 & -0.067 & 0.759 & -0.307 & 0.011 \\
\hline Q15 & 0.018 & 0.689 & -0.223 & 0.044 \\
\hline Q16 & 0.012 & 0.645 & -0.203 & 0.187 \\
\hline Q17 & 0.028 & 0.746 & -0.257 & 0.156 \\
\hline Q18 & 0.384 & 0.326 & 0.178 & 0.297 \\
\hline Q19 & 0.176 & -0.051 & 0.267 & 0.063 \\
\hline Q20 & 0.391 & 0.250 & 0.529 & 0.218 \\
\hline Q21 & 0.415 & 0.178 & 0.367 & 0.478 \\
\hline Q22 & 0.497 & 0.134 & 0.508 & 0.322 \\
\hline Q23 & 0.547 & -0.077 & 0.062 & 0.151 \\
\hline Q24 & 0.293 & 0.487 & 0.425 & -0.435 \\
\hline Q25 & 0.204 & 0.471 & 0.403 & -0.546 \\
\hline Q26 & 0.184 & 0.263 & 0.718 & -0.374 \\
\hline Q27 & 0.203 & 0.170 & 0.720 & -.380 \\
\hline Q28 & 0.370 & 0.271 & 0.262 & 0.116 \\
\hline Q29 & 0.108 & 0.258 & 0.174 & 0.207 \\
\hline Q30 & 0.328 & 0.125 & 0.068 & 0.217 \\
\hline Q31 & 0.579 & 0.210 & 0.287 & 0.383 \\
\hline Q32 & 0.597 & -0.198 & 0.040 & 0.172 \\
\hline
\end{tabular}


al commitment dimension were suggested to be removed.

The Scree plot showed that three or four dimensions could be sufficient for the questionnaire (Figure 1).

\section{Reliability \\ Internal consistency reliability}

The Cronbach's alpha coefficient of final version of the questionnaire was 0.824 . Moreover, Cronbach's alpha coefficient of each dimension of the questionnaire (except professional commitments) was good; Cronbach's alpha of knowledge items was 0.927 , that of attitude items was 0.877 , that of practice items was 0.723 , and that of professional commitments items was 0.449; that was low and showed that the items of professional commitments dimension are not appropriate for the questionnaire.

\section{Test-retest reliability}

Test reliability is measured with a test-retest correlation. Consistency of the retest, according to Spearman coefficient was 0.912 .

\section{Discussion}

With respect to the importance of radiation protection capability amongst operating room nurses and the absence of a proper instrument for measuring radiation protection capability in the operating room field, the present study aimed to develop a radiation protection capability questionnaire.

According to previous studies, radiation risk decreases by using some rules and factors. The most important of these are ALARA principle, the 10 day rule, time, distance, and shielding and awareness about various monitoring devices $[23,34]$. In this questionnaire, the assessment of personnel's awareness of all of these factors is considered.

The face validity, content validity, construct validity, internal homogeneity (Cronbach's alpha), and consistency (test-retest) of the questionnaire were evaluated. So far, there has not been any questionnaire particularly designed for evaluating radiation protection capability in the operating room field, and the available instruments were about limited aspects of ra-

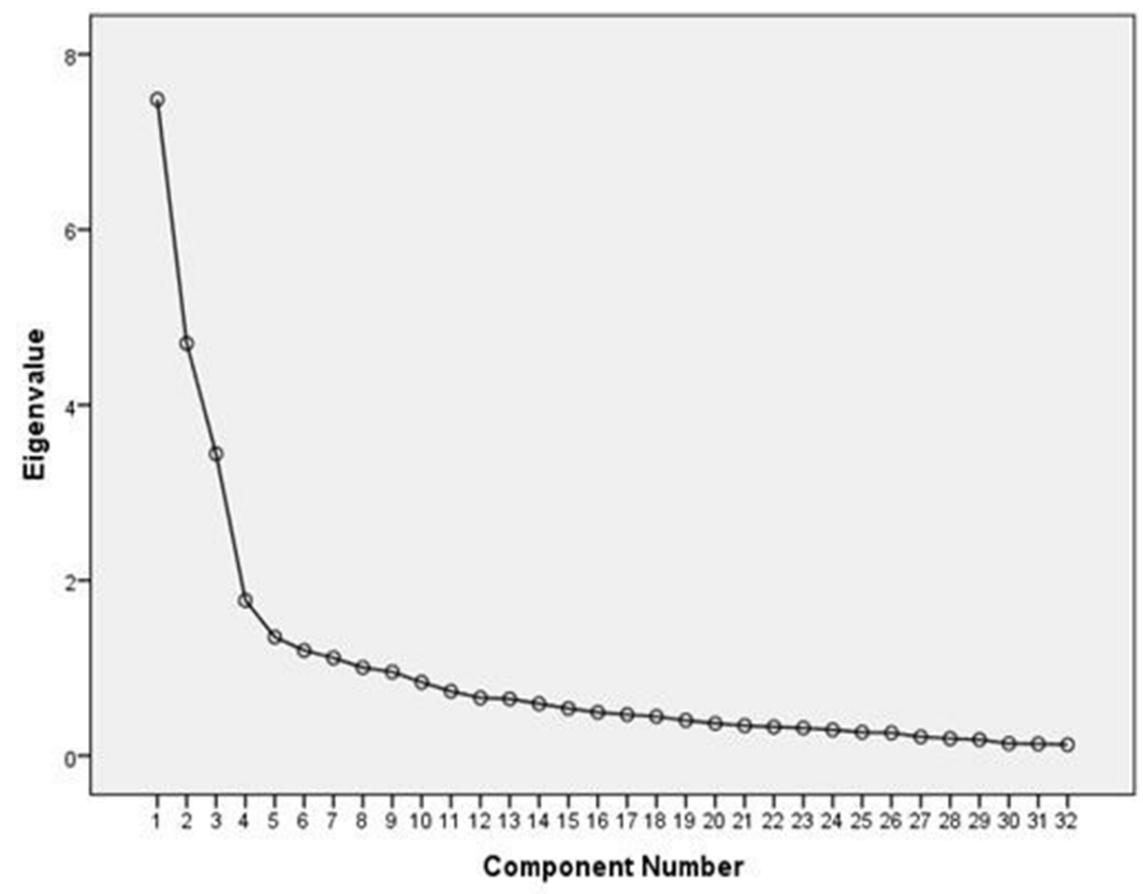

Figure 1: Scree Plot test of factor loading of the items. 
diation protection capability in the operating room [9-13], and no study considered all aspects of radiation protection capability, specific to the operating room field. Moreover, the methods of validity were not confirmed in almost all of the previous studies and only the result of internal homogeneity (Cronbach's alpha) was reported in some of them.

One of the previous questionnaires was designed to evaluate the association of academic education and practical capabilities of radiology technicians in Tabriz University of Medical Science, Iran. This questionnaire was specifically for radiology ward and measured all the duties of a radiology technician, that only one part was radiation protection. Cronbach's alpha coefficient was desirable in mentioned questionnaire but validity had not been verified [13].

Another previous instrument was designed to evaluate the radiation protection knowledge, attitude and practice (KAP) in radiographers and was used in four departments of hospitals, including radiology, operating room, Endoscopic retrograde cholangiopancreatography (ERCP) and Extracorporeal shock wave lithotripsy (ESWL). s-CVR/ Ave of this questionnaire was 0.62 . Other validity methods were not confirmed in this study. Also, reliability was evaluated by test-retest method that the Pearson's correlation coefficient was 0.81 . This instrument was appropriate for radiology ward but was not sufficient and relevant to measure radiation protection in the operating room field [20-22].

Other similar instruments were designed to examine radiation protection knowledge in operating room nurses, that psychometric properties of almost all of these questionnaires were not confirmed $[1,9,11,12]$. Therefore, it was not possible to compare the present questionnaire with previous ones in terms of psychometric properties.

It is obvious that the available questionnaires are not appropriate for evaluating radiation protection capability in the operating room field.

Based on previous researches, evaluating knowledge of radiation protection could be divided into three areas. These areas include radiation physics and radiation biology, radiation protection and guidelines of safe ionizing radiation use [35, 36]. Organizational guidelines and protocols are based on radiation physics and radiation protection principles under standard radiation conditions. These protocols are improved and upgraded by a medical physicist known as radiation safety officer (RSO) according to the conditions in each hospital. Therefore, knowledge of protocols and their observance will have the greatest contribution in reducing radiation damage to staff and patients. Therefore, the number of questions related to guidelines and protocols is considered more than other questions in this questionnaire.

Developing and evaluating the psychometric characteristics of a questionnaire about radiation protection capability of operating room nurses were confirmed in this study. It should be noted that unlike previous studies, patients' radiation protection is highlighted in this questionnaire.

One limitation of this study was that the radiation exposure condition was not the same in different operating rooms. Besides, different countries may have their own national guidelines and regulations.

Therefore, further studies with larger numbers of participants, different kind of operating rooms and across different countries are needed to be conducted.

Based on the results, this questionnaire is a new valid and reliable instrument that could comprehensively evaluate the radiation protection capability of operating room nurses.

\section{Conclusion}

In the present study, a questionnaire was designed in Iran for measuring radiation protection capability of operating room nurses. The results of the study show that the psychomet- 
Questionnaire of Radiation Protection Capability

ric properties of the questionnaire are satisfactory. This psychometric questionnaire can be used in the development of programs for solving radiation protection problems in the operating rooms. By utilizing this questionnaire, educational and management needs can be estimated.

\section{Acknowledgment}

The authors would like to thank all the experts for their scientific advice, and all the operating room nurses, who voluntarily participated in this study. The authors wish to thank Mr. H. Argasi at the Research Consultation Centre (RCC) of Shiraz University of Medical Sciences for his invaluable assistance in editing this manuscript.

\section{Conflict of Interest}

None

\section{References}

1. Jentzsch T, Pietsch CM, Stigler B, Ramseier LE, Seifert B, Werner CM. The compliance with and knowledge about radiation protection in operating room personnel: a cross-sectional study with a questionnaire. Arch Orthop Trauma Surg. 2015;135(9):1233-40. doi: 10.1007/s00402-0152257-z. PubMed PMID: 26088030.

2. Ramos M, Montoro A, Almonacid M, Ferrer S, Barquinero J F, Tortosa R, Verdú G, Rodríguez P,Barrios LL, Villaescusa Jl. Radiation effects analysis in a group of interventional radiologists using biological and physical dosimetry methods. Eur J Radiol. 2010;75(2):259-64. doi: 10.1016/j. ejrad.2009.03.035. PubMed PMID: 19380209.

3. Roguin A, Goldstein J, Bar O, Goldstein J. Brain and Neck Tumors Among Physicians Performing Interventional Procedures. The American Journal of Cardiology. 2013;111(9):1368-72. doi: 10.1016/j.amjcard.2012.12.060.

4. Lim Y, Byun HJ, Park CS, Lee JH, Park J, Lee $\mathrm{JH}$. Primary cutaneous carcinosarcoma developing after chronic C-arm radiation exposure. JAAD Case Rep. 2018;4(2):126-8. doi: 10.1016/j. jdcr.2017.07.024. PubMed PMID: 29387761. PubMed PMCID: PMC5789523.

5. Anupam M. Occupational Radiation Exposure from C Arm Fluoroscopy During Common Orthopaedic Surgical Procedures and its Pre- vention. J Clin Diagn Res. 2015;9(3):1-4. doi: 10.7860/JCDR/2015/10520.5672. PubMed PMID: 25954671. PubMed PMCID: PMC4413121.

6. Hyun SJ, Kim KJ, Jahng TA, Kim HJ. Efficiency of lead aprons in blocking radiation - how protective are they? Heliyon. 2016;2(5):1-14. doi: 10.1016/j. heliyon.2016.e00117. PubMed PMID: 27441288. PubMed PMCID: PMC4946218.

7. Do H-K. General Principles of Radiation Protection in Fields of Diagnostic Medical Exposure. $J$ Korean Med Sci. 2016;31:S6-9. doi: 10.3346/ jkms.2016.31.S1.S6. PubMed PMID: 26908991. PubMed PMCID: PMC4756345.

8. Ansari A. Kleinhans K. Boice JD. Potential health effects of low dose radiation and what it means to the practice of radiation protection. $J$ Radiol Prot. 2019;39(4):E9-13. doi: 10.1088/1361-6498/ ab3fea. PubMed PMID: 31756172

9. Tunçer N, Kuyucu E, Sayar S, Polat G, Erdil I, Tuncay I. Orthopedic surgeons' knowledge regarding risk of radiation exposition: a survey analysis. S/COT J. 2017;3:29. doi: 10.1051/sicotj/2017008. PubMed PMID: 28387195. PubMed PMCID: PMC5384318.

10. Babaloui S, Parwaie W, Refahi S, Abrazeh M, AfkhamiArdekani M. Awareness Assessment of Nurses in the OR, ICU, CCU, and PICU About Radiation Protection Principles of Portable Radiography in Hospitals of Bandar Abbas, Iran. Journal of Radiology Nursing. 2018;37(2):126-9. doi: 10.1016/j.jradnu.2017.12.005.

11. Tefera E, Qureshi SA, Gezmu AM, Mazhani L. Radiation protection knowledge and practices in interventional cardiologists practicing in Africa: a cross sectional survey. J Radiol Prot. 2020;40(1):311-8. doi: 10.1088/1361-6498/ab5840. PubMed PMID: 31731286 .

12. Tok A, Akbas A, Aytan N, Aliskan T. Are the urology operating room personnel aware about the ionizing radiation? Int Braz J Urol. 2015;41(5):982-9. doi: 10.1590/S1677-5538.IBJU.2014.0351.

13. Farajollahi A, Ghojazadeh M, Movaffagi A, Alikhani $H$. Association of academic education and practical capabilities of radiology technologists. J Anal Res Clin Med. 2015;3(1):23-9. doi: 10.15171/ jarcm.2015.004

14. Reagan JT. Slechta AM. Factors related to radiation safety practices in California. Radiol Technol. 2010;81(6):538-47. PubMed PMID: 20606048.

15. Ferzer RL. Capability and clinical success. The Annals of Family Medicine Neonatal Nursing. Ann Fam Med. 2010;8(5):454-60. doi: 10.1370/ afm.1163. PubMed PMID: 20843888. PubMed 
PMCID: PMC2939422.

16. Fiebich M. Practical radiation protection of the patient in radiological diagnostics. Radiologe. 2017;57(7):534-40. doi: 10.1007/s00117-0170258-3. PubMed PMID: 28523491.

17. Faggioni L, Paolicchi F, Bastiani L, Guido D, Caramella $D$. Awareness of radiation protection and dose levels of imaging procedures among medical students, radiography students, and radiology residents at an academic hospital: Results of a comprehensive survey. Eur J Radiol. 2017;86:135-42. doi: 10.1016/j.ejrad.2016.10.033. PubMed PMID: 28027740.

18. Hobbs JB, Goldstein N, Lind KE, Elder D, Dodd GD, Borgstede JP. Physician Knowledge of Radiation Exposure and Risk in Medical Imaging. $J$ Am Coll Radiol. 2018;15(1):34-43. doi: 10.1016/j. jacr.2017.08.034. PubMed PMID: 29100884.

19. Yurt A, Çavuşoğlu B, Günay T. Evaluation of Awareness on Radiation Protection and Knowledge About Radiological Examinations in Healthcare Professionals Who Use Ionized Radiation at Work. Mol Imaging Radionucl Ther. 2014;23(2):48-53. doi: 10.4274/mirt.00719. PubMed PMID: 24963445. PubMed PMCID: PMC4067876.

20. Moshfegh SH, Hasanzadeh H, Jadidi M, Mirmohammadkhani M. Evaluation of Knowledge, Attitude and Practice of Personnel in Operating Room, ERCP, and ESWL Towards Radiation Hazards and Protection. Middle East J Rehabil Health Stud. 2017;4(3):1-6. doi: 10.5812/mejrh.12354.

21. Shabani M, Hasanzadeh H, Emadi A, Mirmohammadkhani M. Radiation Protection Knowledge, Attitude, and Practice (KAP) in Interventional Radiology. Oman Med J. 2018;33(2):141-7. doi: 10.5001/omj.2018.26. PubMed PMID: 29657683. PubMed PMCID: PMC5889843.

22. Masoumi H, Hasanzadeh H, Jadidi M, Mirmohammadkhani M. A Survey on the Radiation Protection Status among Radiology Staff. Iran J Med Phys. 2018;15(3):176-82. doi: 10.22038/ IJMP.2018.24725.1249.

23. Hirvonen L, Schroderus-Salo T, Henner A, Ahonen S, Kääriäinen M. Miettunen J, Mikkonen K. Nurses' knowledge of radiation protection: A cross-sectional study. Radiography. 2019;25(4):e108-12. doi: 10.1016/j.radi.2019.04.011.

24. Lakhwani OP, Dalal V, Jindal M, Nagala A. Radiation protection and standardization. J Clin Orthop Trauma. 2019;10(4):738-43. doi: 10.1016/j. jcot.2018.08.010. PubMed PMID: 31316247. PubMed PMCID: PMC6611844.
25. Magrini SM, Pasinetti N, Belgioia L, Triggiani L, Levis M, Ricardi U, Renzo Corvò. Applying radiation protection and safety in radiotherapy. Radiol Med. 2019;124(8):777-82. doi: 10.1007/s11547019-01043-7. PubMed PMID: 31104276.

26. Aljabal AF, Wargo RR, Lin PP. Evaluation of lead equivalence of radiation protection apparatuses as a function of tube potential and spectral shaping filter. J Appl Clin Med Phys. 2019;20(12):204-9. doi: 10.1002/acm2.12768.

27. Wojcik A, Harms-Ringdah M. Radiation protection biology then and now. Int $J$ Radiat Biol. 2019;95(7):841-50. doi: 10.1080/09553002.2019.1589027. PubMed PMID: 30831044.

28. Matthews EP. Radiation Physics, Biology, and Protection. Radiol Technol. 2019;90(5):471-85. PubMed PMID: 31088948.

29. Mousazadeh S, Rakhshan M, Mohammadi F. Investigation of Content and Face Validity and Reliability of sociocultural Attitude towards Appearance Questionnaire-3 (SATAQ-3) among Female Adolescents. Iran J Psychiatry. 2017;12(1):15. PubMed PMID: 28496497.

30. Lawshe $\mathrm{CH}$. A quantitative approach to content validity. Pers Psychol. 1975;28(4):563-75. doi: 10.1111/j.1744-6570.1975.tb01393.x.

31. Waltz CF. Validation study. NLN Publ. 1988;(182249):155-62. PubMed PMID: 3412881.

32. Torabizadeh C, Yousefinya A, Zand F, Rakhshan M, Fararooei M. A nurses' alarm fatigue questionnaire: development and psychometric properties. J Clin Monit Comput. 2017;31(6):1305-12. doi: 10.1007/ s10877-016-9958-X. PubMed PMID: 27848141.

33. Burns N, Grove SK. Understanding nursing research: building an evidence-based practice. 5th ed. Philadelphia: Elsevier Saunders; 2010.

34. Bushong S C. Radiologic science for technologistsE-book: physics, biology, and protection. Elsevier Health Science; 2013.

35. Salah Eldeen NG, Farouk SA. Assessment of Awareness and Practice of Ionizing Radiation Protection Procedures among Exposed Health Care Workers. Egyptian Journal of Occupational Medicine. 2020;44(1):529-44. doi: 10.21608/ EJOM.2020.67771.

36. Schroderus-Salo T, Hirvonen L, Henner A, Ahonen S, Kääriäinen M, Miettunen J, Mikkonen K. Development and validation of a psychometric scale for assessing healthcare professionals' knowledge in radiation protection. Radiography. 2019;25(2):13642. doi: 10.1016/j.radi.2018.12.010. 